\title{
Upwinding in finite element systems of differential forms
}

\author{
Snorre H. Christiansen* \\ Published as the Smale Prize Lecture in: \\ Foundations of computational mathematics, Budapest 2011, \\ London Mathematical Society Lecture Note Series, 403, \\ Cambridge University Press, 2013.
}

\begin{abstract}
We provide a notion of finite element system, that enables the construction spaces of differential forms, which can be used for the numerical solution of variationally posed partial differential equations. Within this framework, we introduce a form of upwinding, with the aim of stabilizing methods for the purposes of computational fluid dynamics, in the vanishing viscosity regime.
\end{abstract}

\section{Foreword}

I am deeply honored to receive the first Stephen Smale prize from the Society for Foundations of Computational Mathematics.

I want to thank the jury for deciding, in what I understand was a difficult weighting process, to tip the balance in my favor. The tiny margins that similarly enable the Gömböc to find its way to equilibrium, give me equal pleasure to contemplate. It's a beautiful prize trophy.

It is a great joy to receive a prize that celebrates the unity of mathematics. I hope it will draw attention to the satisfaction there can be, in combining theoretical musings with potent applications. Differential geometry, which infuses most of my work, is a good example of a subject that defies perceived boundaries, equally appealing to craftsmen of various trades.

As I was entering the subject, rumors that Smale could turn spheres inside out without pinching, were among the legends that gave it a sense of surprise and mystery. I also remember reading about Turing machines built on other rings than $\mathbb{Z} / 2 \mathbb{Z}$, which, together with parallelism and quantum computing, convinced me that the foundations of our subject were still in the making. Happy for the occasion provided by the FoCM conference, to meet the master, I was also a bit intimidated to learn that we have a common interest in discrete de Rham sequences. They are the topic of this paper.

Many people have generously shared their insights and outlooks with me. I feel particularly indebted, mathematically as well as personally, to Jean-Claude

${ }^{*}$ Centre of Mathematics for Applications \& Department of Mathematics, University of Oslo, PO Box 1053 Blindern, NO-0363 Oslo, Norway 
Nédélec, Annalisa Buffa and Ragnar Winther. I'd like to dedicate this paper to my grandmother, who will probably joke, as she usually does, that she has noticed some mistakes on page 5 . She constitutes an important fraction of my readership, also numberwise.

I'm very grateful for this opportunity to make my work more widely known.

\section{Introduction}

Finite elements come in a variety of brands. The particular flavour considered here, called mixed finite elements, was pioneered by [36, 34] and has since developed into versatile tool for the numerical solution of a variety of partial differential equations describing, for instance, fluid flow and electromagnetic wave propagation [9, 37, 31]. As remarked in [7], lowest order elements correspond to constructs in algebraic topology referred to as Whitney forms [44, 45, 23]. Uniting these strands has led to the topic of finite element exterior calculus $[27,2,28,15,3]$, most recently reviewed in [5].

Finite element systems (FES) were introduced in [16] to provide a generalization to arbitrary dimension of the dual elements constructed in [14]. This general framework allows for a unified analysis of the preceding mixed finite elements, but can also accommodate polyhedral decompositions of spaces (rather than just simplices and products thereof) as well as general differential forms (rather than just polynomial ones). This paper contains an introduction to FES, referring to $[17,20]$ for more ample treatments. The flexibility of FES with respect to meshing techniques is already quite standard in mimetic finite differences and finite volumes, with which one observes a confluence of techniques [11, 6, 24]. For our present purposes it is, however, the flexibility with respect to choice of local functions that is of interest, since we will need exponentials and variants thereof.

In spite of the topological twist of the subject, which might remind of [25, 8], the "upwinding" of the title refers not to winding numbers but to wind, of the blowing kind. The equations of fluid dynamics typically feature a convective term, hyperbolic in nature, moderated by a diffusive one, elliptic in nature. As the viscosity vanishes, the nature of the equations changes, say from NavierStokes to Euler's equations, in what is called a singular limit. The highest order derivatives are eliminated in a delicate limiting process, where fields display sharp gradients and form boundary layers.

To handle this convection dominated regime, which is important for many applications, special numerical methods have been designed, often under the name upwinding, for instance the famous [13]. They achieve stability by taking into account the direction in which quantities (such as fluid densities or momentum) are transported [32,38]. This paper is devoted to how a form of upwinding, extending $[1,41,12,46]$, can be incorporated in an FES, expanding upon Example 5.31 in [20].

Section 1 serves as an appetizer, giving an introduction to the finite element method in dimension one, exemplifying problems and solutions related to vanishing viscosity. Section 2 gives some ingredients on discrete geometry useful for the definition of finite element systems, which is provided in 3. In section 4 we serve the upwinding technique which we have cooked up for FES. Section 5 contains some remarks for further rumination. 


\section{Upwinding in dimension one}

In this section we sketch a numerical method for solving one-dimensional convectiondiffusion problems, which is adapted to the convection dominated regime. This well known method goes back at least to $[1,41]$ but is presented here in a finite element language, as in e.g. [33]. Our numerical illustrations follow [39] quite closely. This will give a quick introduction to the finite element method and motivate the generalizations presented in the following sections.

We choose $a<b$ in $\mathbb{R}$ and denote by $I$ the interval $[a, b]$. For given $\alpha>0$ (viscosity), $\beta \in \mathbb{R}$ (convection) and $f: I \rightarrow \mathbb{R}$ (source) we want to solve the second order differential equation for $u: I \rightarrow \mathbb{R}$ :

$$
\begin{array}{r}
-\alpha u^{\prime \prime}+\beta u^{\prime}=f, \\
u(a)=0 \text { and } u(b)=0 .
\end{array}
$$

This problem can be given a variational formulation. Let $X$ denote the space:

$$
X=\mathrm{H}_{0}^{1}(I)=\left\{u \in \mathrm{L}^{2}(I): u^{\prime} \in \mathrm{L}^{2}(I), u(a)=0, u(b)=0\right\} .
$$

The scalar product of $\mathrm{L}^{2}(I)$ and its extensions by continuity to dualities between Sobolev spaces, will be denoted:

$$
\langle u, v\rangle=\int u v
$$

We denote by $A$ the differential operator:

$$
A: u \mapsto-\alpha u^{\prime \prime}+\beta u^{\prime}
$$

and define a bilinear form $a$ on $X$ by:

$$
a(u, v)=\langle A u, v\rangle=\alpha \int u^{\prime} v^{\prime}+\int \beta u^{\prime} v
$$

Introduce also a linear form $l$ on $X$ defined by:

$$
l(v)=\langle f, v\rangle=\int f v .
$$

The variational formulation of the above problem $(1.1,1.2)$ is:

$$
u \in X, \forall v \in X \quad a(u, v)=l(v) .
$$

We now turn to the discretization of (1.8). Given a positive $n \in \mathbb{N}$ we choose points $x_{i} \in[a, b]$ for $i \in[[0, n]$, such that:

$$
a=x_{0}<\cdots<x_{i}<x_{i+1}<\cdots<x_{n}=b .
$$

These points subdivide $[a, b]$ into $n$ intervals of the form $\left[x_{i}, x_{i+1}\right]$ and we denote by $h_{n}$ the length of the longest one:

$$
h_{n}=\max \left\{\left|x_{i+1}-x_{i}\right|: i \in[[0, n-1]]\right\} .
$$

We let $X_{n}$ denote the subspace of $X$ consisting of functions that are piecewise affine with respect to this subdivision. The Galerkin discretization of (1.8) is:

$$
u_{n} \in X_{n}, \forall v \in X_{n} \quad a\left(u_{n}, v\right)=l(v) .
$$


For the purposes of analysis, one considers a sequence of such subdivisions, indexed by $n$, providing a sequence $\left(u_{n}\right)$ of approximate solutions to (1.8). For fixed $\alpha>0, \beta \in \mathbb{R}, f \in \mathrm{L}^{2}(I)$ one has convergence of $\left(u_{n}\right)$ to $u$, for instance in the sense:

$$
\left\|u-u_{n}\right\|_{\mathrm{L}^{2}(I)} \leq C h_{n}^{2} \text { and }\left\|u^{\prime}-u_{n}^{\prime}\right\|_{\mathrm{L}^{2}(I)} \leq C^{\prime} h_{n} .
$$

Of course the constants $C, C^{\prime}$ depend on $\alpha, \beta, f$. In practice we are interested in a regime where $\beta$ and $f$ are moderate ("of order 1"), whereas $\alpha$ is several magnitudes smaller. One analyses this regime by fixing $\beta \neq 0$ and $f$, and considering the asymptotic behavior of the sequence $u_{n}[\alpha]$, when $\alpha \rightarrow 0$ and $n \rightarrow \infty$. This is also referred to as the vanishing viscosity limit.

For illustration we chose $a=-1, b=1, \beta=1$ and $f=1$. The exact solutions $u[\alpha]$ for various $\alpha$ are plotted in the top graph in Figure 1. As $\alpha \rightarrow 0$ the solution approaches the function $u[0]: I \rightarrow \mathbb{R}$ which solves:

$$
\begin{aligned}
\beta u^{\prime} & =f, \\
u(a) & =0 .
\end{aligned}
$$

Notice that of the two boundary conditions (1.2), only one is retained by the limit $u[0]$. At the other boundary point the small viscosity solutions $u[\alpha]$ display singular behavior, with formation of a boundary layer. Given that $\alpha>$ 0 , the part of the boundary where the homogeneous boundary condition is respected, is the one where $\beta$, interpreted as a vectorfield on $I$, points into the domain, called the inflow boundary ( $a$ in our case). The boundary layer appears where $\beta$ points out of the domain, called the outflow boundary ( $b$ in our case). The thickness of this layer is roughly $\alpha / \beta$. Since the differential equation degenerates in its highest order term, this is an example of what is called a singular perturbation problem, for which the above (singular) behavior can be said to be typical.

For the Galerkin discretizations we have chosen equispaced points with $n=25$. Results are plotted in the middle row of Figure 1 . As the viscosity $\alpha$ approaches 0 , the quality of the numerical solution deteriorates, with apparition of unwanted oscillations, extending far beyond the boundary layer. The numerical stability is often evaluated in terms of the dimensionless so-called Péclet number:

$$
\mathrm{Pé}=h_{n} \beta / \alpha .
$$

In the numerical experiments, $\alpha$ was chosen in such a way that:

$$
\text { Pé }=10^{k}, k \in\{-0.5,0,0.5,1\} .
$$

The graphs illustrate that the numerical method rapidly deteriorates when Pé increases above 1 . Notice that the problem is related to stability rather than consistency: at least away from the outflow boundary, the Galerkin space $X_{n}$ contains rather good approximations of the exact solution, but the method is bad at choosing one.

A better numerical method can be obtained by cleverly choosing a new space $Y_{n}$ and solving:

$$
u_{n} \in X_{n}, \forall v \in Y_{n} \quad a\left(u_{n}, v\right)=l(v) .
$$

Such methods are called Petrov-Galerkin methods. One distinguishes between the trial space $X_{n}$ and the test space $Y_{n}$. A rationale for the choice of $Y_{n}$ can be 

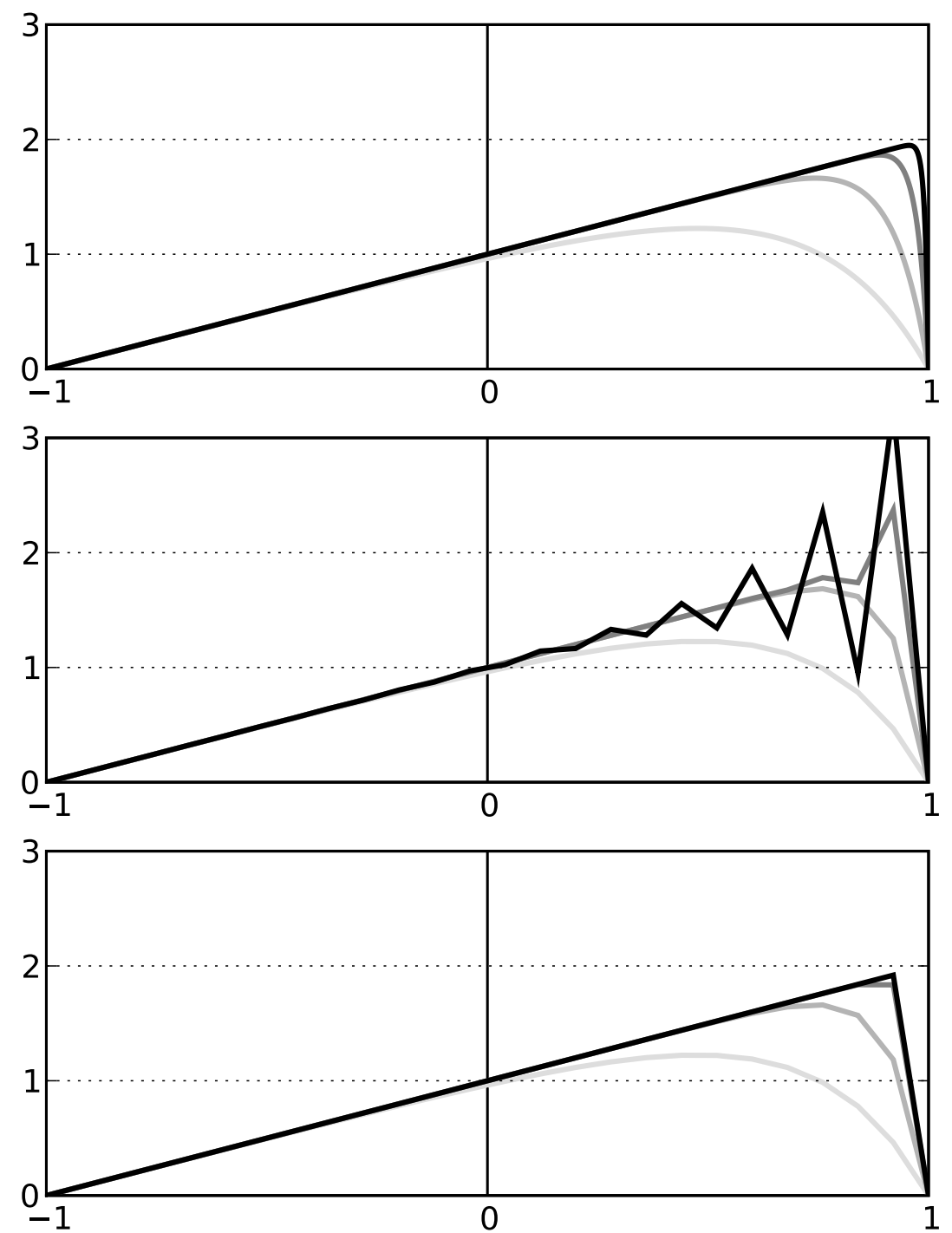

Figure 1: Decreasing viscosity from light gray to black. Exact solutions (top), standard piecewise affine finite elements (middle), affine trial functions and upwinded test functions (bottom). 
obtained as follows. Choose a scalar product $b$ on $X$. Let $a^{\star}$ be the transpose of $a$, the bilinear form associated with the adjoint differential operator:

$$
A^{\star}: v \mapsto-\alpha v^{\prime \prime}-\beta v^{\prime} .
$$

Denote by $\Phi: X \rightarrow X$ the map determined by solving the adjoint problems:

$$
\forall v, w \in X \quad a^{\star}(\Phi(w), v)=b(w, v) .
$$

If we now solve (1.17) with $Y_{n}=\Phi\left(X_{n}\right)$ we notice the following. For all $w \in X_{n}$ :

$$
b\left(w, u_{n}\right)=a\left(u_{n}, \Phi(w)\right)=l(\Phi(w))=a(u, \Phi(w))=b(w, u) .
$$

Therefore $u_{n}$ is the $b$-orthogonal projection of $u$ on $X_{n}$. Orthogonal projection, which yields the best approximation with respect to the chosen norm, is typically a more stable procedure than the Galerkin projection associated with non-symmetric bilinear forms, such as $a$.

However this method can seem unpractical : for a given basis $\left(e_{i}\right)$ of $X_{n}$ it might seem too difficult a task to determine the basis $f_{i}=\Phi\left(e_{i}\right)$ of $Y_{n}$. Recall that the canonical basis of $X_{n}$ consists of the functions $\lambda_{i}: I \rightarrow \mathbb{R}$, indexed by $i \in\left[[1, n-1]\right.$, uniquely determined by the properties $\lambda_{i} \in X_{n}$ and $\lambda_{i}\left(x_{j}\right)=\delta_{i j}$ (Kronecker $\delta$ ) for all $j \in[\llbracket 1, n-1]$. As it turns out, for certain choices of $b$ we can construct a similar basis for $Y_{n}$ quite easily, providing not a free lunch but a bargain one.

Let $b$ be defined by:

$$
b(v, w)=\int v^{\prime} w^{\prime}=\langle B v, w\rangle .
$$

We notice that $B: v \mapsto-v^{\prime \prime}$ induces an isomorphism:

$$
B: X_{n} \rightarrow Z_{n}=\underset{i \in \llbracket 1, n-1 \rrbracket}{\bigoplus} \mathbb{R} \delta_{x_{i}},
$$

with Dirac $\delta$ 's at interior vertices. A basis $\left(\lambda_{i}^{\star}\right)$ of $Y_{n}=\Phi\left(X_{n}\right)=A^{\star-1} B\left(X_{n}\right)$ can be obtained by choosing $\lambda_{i}^{\star}: I \rightarrow \mathbb{R}$ to be the unique function satisfying $\lambda_{i}^{\star}\left(x_{j}\right)=\delta_{i j}$ for all $j \in[[1, n-1]]$ (as for the standard basis) and :

$$
\left.A^{\star} \lambda_{i}^{\star}=0 \text { on }\right] x_{j}, x_{j+1}[\text { for each } j \in[[0, n-1]] .
$$

Indeed $A^{\star}$ then induces an isomorphism:

$$
A^{\star}: Y_{n} \rightarrow Z_{n} .
$$

The basis functions $\lambda_{i}^{\star}$ can be determined explicitly. When $\beta=0$, the definition (1.23) yields the standard basis. For $\beta \neq 0, \lambda_{i}^{\star}$ is, on each sub-interval, a linear combination of a constant and an exponential:

$$
\lambda_{i}^{\star}(x)=c_{0}+c_{1} \exp (r x) \text { with } r=-\beta / \alpha, \text { and } c_{0}, c_{1} \in \mathbb{R} .
$$

For $r=0$ one should replace the above definition by affine functions. Typical basis functions are represented in Figure 2 for different values of $r$. Actually the chosen values are:

$$
r=10^{k}-10^{-k} \text { for } k \in\{0.5,0,-0.5,-1,-1.5\} .
$$




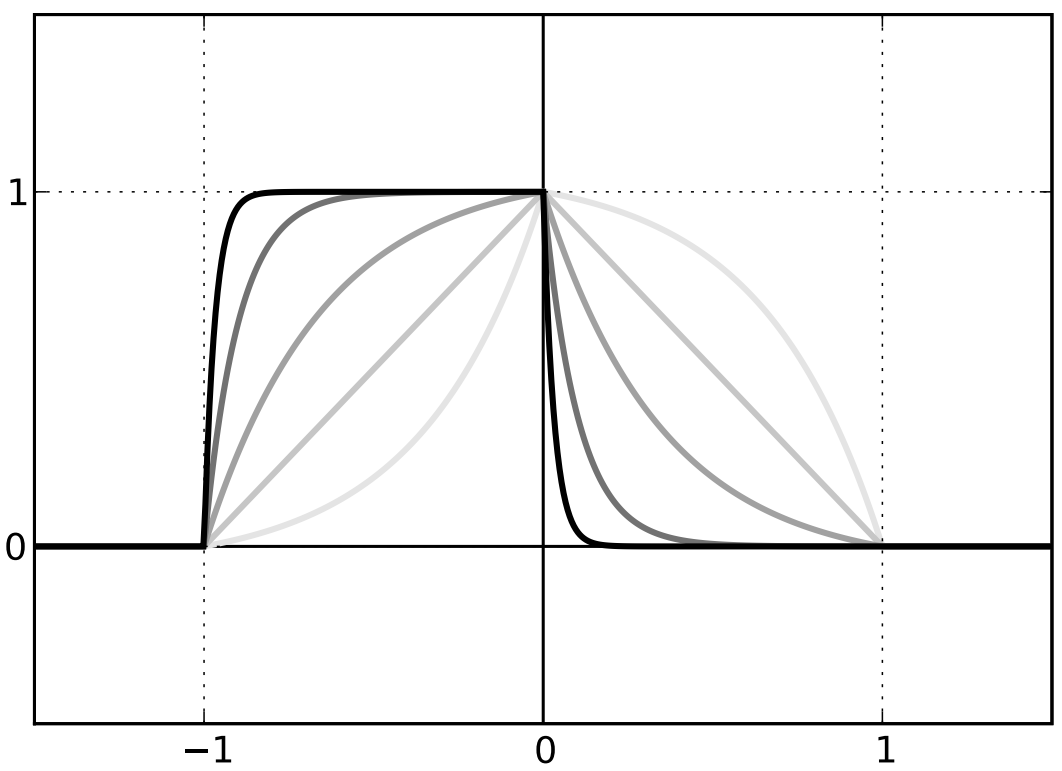

Figure 2: Basis functions, from moderately downwinded (light gray) to strongly upwinded (black). Wind blowing from left to right.

In the bottom row of Figure 1 the numerical solutions for the Petrov-Galerkin method (1.17) with upwinded basis functions for $Y_{n}$ are plotted.

There are many senses in which, as $\alpha \rightarrow 0$, the solution $u[\alpha]$ converges to $u[0]$ - but the norm associated with (1.21) is not one of them. In fact:

$$
\|u[\alpha]\|_{b} \rightarrow \infty \text { as } \alpha \rightarrow 0,
$$

precluding even weak convergence in $X$. This indicates that the $b$-norm is a poor choice for convergence analysis of the numerical method in the vanishing viscosity regime. From this point of view it is also a bit paradoxical that the numerical results are so satisfactory. A better, but $\alpha$-dependent, norm is defined by:

$$
b[\alpha](v, w)=\alpha \int v^{\prime} w^{\prime}+\int v w,
$$

for which one can check:

$$
\|u[\alpha]-u[0]\|_{b[\alpha]} \rightarrow 0 \text { as } \alpha \rightarrow 0 .
$$

For the numerics we can use the approximation:

$$
\begin{gathered}
b[\alpha, n](v, w)=\alpha \int v^{\prime} w^{\prime}+\sum_{i} v\left(x_{i}\right) w\left(x_{i}\right) \mu_{i}, \\
\text { with } \mu_{i}=\left(x_{i+1}-x_{i-1}\right) / 2 .
\end{gathered}
$$

With this choice we still have an isomorphism (1.22) so we get the same numerical method as for (1.21). 
Coincidentally the choice $b[0, n]$ shows that the Petrov-Galerkin solution $u_{n}[\alpha]$ is in fact the element of $X_{n}$ interpolating $u[\alpha]$ at vertices. We conclude that it is time to go to higher dimensions. We retain that the finite element method divides space (the interval) into cells, defines functions locally on these cells (with polynomials or exponentials for instance), imposing mere continuity between cells. Stability can be achieved by creating pairs of spaces where local functions match, through the differential operators of the problem to solve.

\section{Discrete geometry}

Useful references for the considerations recalled in this section include $[25,8]$.

\subsection{Cellular complexes}

For any natural number $k \geq 1$, let $\mathbb{B}^{k}$ be the closed unit ball of $\mathbb{R}^{k}$ and $\mathbb{S}^{k-1}$ its boundary. For instance $\mathbb{S}^{0}=\{-1,1\}$. We also put $\mathbb{B}^{0}=\{0\}$.

Let $S$ denote a compact metric space. A $k$-dimensional cell in $S$ is a closed subset $T$ of $M$ for which there is a Lipschitz bijection $\mathbb{B}^{k} \rightarrow T$ with a Lipschitz inverse (if a cell $T$ is both $k$ - and $l$-dimensional then $k=l$ ). For $k \geq 1$, we denote by $\partial T$ its boundary, the image of $\mathbb{S}^{k-1}$ by the chosen bi-Lipschitz map (different such maps give the same boundary). The interior of $T$ is by definition $T \backslash \partial T$ (it is open in $T$ but not necessarily in $S$ ).

Definition 2.1. A cellular complex is a pair $(S, \mathcal{T})$ where $S$ is a compact metric space and $\mathcal{T}$ is a finite set of cells in $S$, such that the following conditions hold:

- Distinct cells in $\mathcal{T}$ have disjoint interiors.

- The boundary of any cell in $\mathcal{T}$ is a union of cells in $\mathcal{T}$.

- The union of all cells in $\mathcal{T}$ is $S$.

The subset of $\mathcal{T}$ consisting of $k$-dimensional cells is denoted $\mathcal{T}^{k}$.

$$
\mathcal{T}^{k}=\{T \in \mathcal{T}: \operatorname{dim} T=k\} .
$$

We also say that $\mathcal{T}$ is a cellular complex on $S$.

Example 2.1. Choose $a<b$ in $\mathbb{R}$ and let $S=[a, b]$. Choose points $x_{i} \in[a, b]$ for $i \in[[0, n]]$, such that:

$$
a=x_{0}<\cdots x_{i}<x_{i+1} \cdots<x_{n}=b .
$$

The following determines a cellular complex on $S$ :

$$
\begin{aligned}
& \mathcal{T}^{0}=\left\{x_{i}: i \in[[0, n]\},\right. \\
& \mathcal{T}^{1}=\left\{\left[x_{i}, x_{i+1}\right]: i \in[[0, n-1]]\right\}, \\
& \mathcal{T}=\mathcal{T}^{0} \cup \mathcal{T}^{1}
\end{aligned}
$$

In fact all cellular complexes on $S$ are of this form, for a uniquely determined choice of points $x_{i}$ subject to (2.2). 


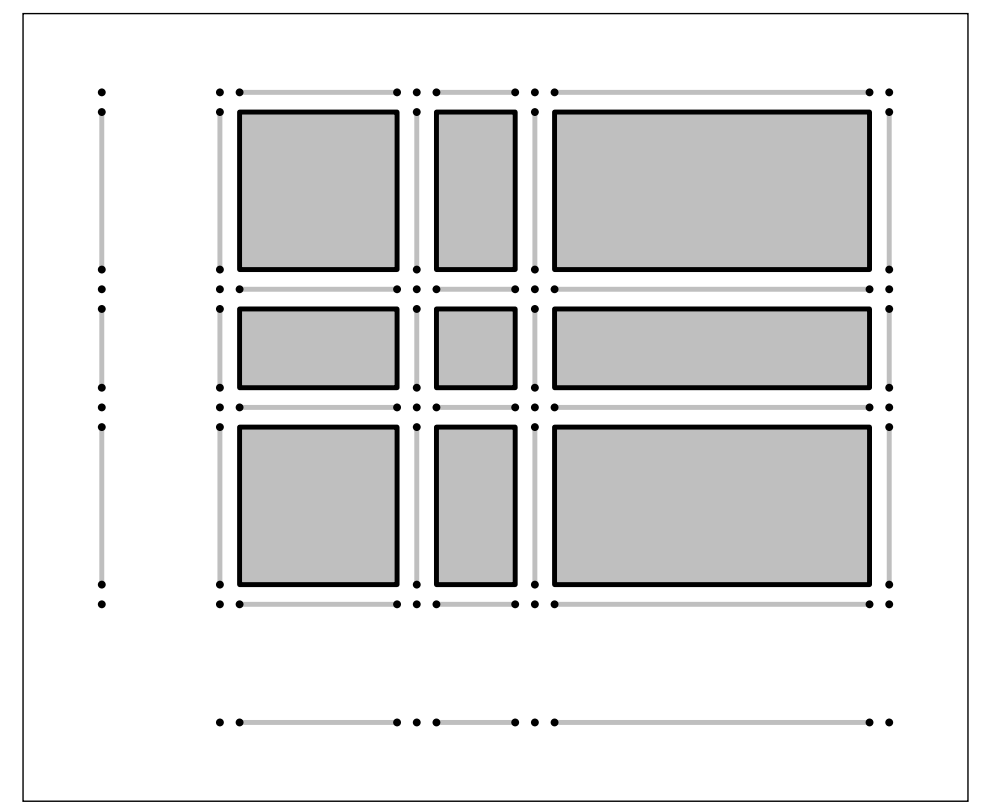

Figure 3: Product complex of two one-dimensional complexes. Cells are represented in gray, with black boundaries.

Example 2.2. Suppose we have two compact metric spaces $M$ and $N$, equipped with cellular complexes $\mathcal{U}$ and $\mathcal{V}$. On $M \times N$ we let $\mathcal{U} \times \mathcal{V}$ denote the product cellular complex on $M \times N$, whose cells are all those of the form $U \times V$ for some $U \in \mathcal{U}$ and $V \in \mathcal{V}$.

This construction is illustrated in Figure 3 for the product of two intervals equipped with cellular complexes.

The boundary $\partial T$ of any cell $T$ of $\mathcal{T}$ can be naturally equipped with a cellular complex, namely:

$$
\left\{T^{\prime} \in \mathcal{T}: T^{\prime} \subseteq T \text { and } T^{\prime} \neq T\right\} .
$$

We use the same notation for the boundary of a cell and the cellular complex it carries.

A refinement of a cellular complex $\mathcal{T}$ on $S$ is a cellular complex $\mathcal{T}^{\prime}$ on $S$ such that each element of $\mathcal{T}$ is the union of elements of $\mathcal{T}^{\prime}$. We will be particularly interested in simplicial refinements of cellular complexes.

A cellular subcomplex of a cellular complex $\mathcal{T}$ on $S$, is a cellular complex $\mathcal{T}^{\prime}$ on some closed part $S^{\prime}$ of $S$ such that $\mathcal{T}^{\prime} \subseteq \mathcal{T}$. For instance if $T \in \mathcal{T}$ is a cell, its subcells form a subcomplex of $\mathcal{T}$, which we denote by $\tilde{T}$. We have seen that the boundary of any cell $T \in \mathcal{T}$ can be equipped with a cellular complex which is a subcomplex of $\tilde{T}$.

A simplicial complex on a set $S$, is a set $\mathcal{S}$ of non-empty finite subsets of $S$, such that for all $T$ in $\mathcal{S}$ the non-empty subsets of $T$ are also in $\mathcal{S}$. The elements of $\mathcal{S}$ are called simplices, the elements of a simplex vertices and the non-empty subsets of a simplex faces.

We attach a reference cell to any simplex $T \in \mathcal{S}$ as follows:

$$
|T|=\left\{x=\left(x_{i}\right)_{i \in T} \in \mathbb{R}^{T}: \sum_{i \in T} x_{i}=1 \text { and } \forall i \in T \quad x_{i} \geq 0\right\} \text {. }
$$


If $T^{\prime} \subseteq T$ we have a canonical affine map $i_{T T^{\prime}}:\left|T^{\prime}\right| \rightarrow|T|$. The direct limit of this system is denoted $|\mathcal{S}|$. It is obtained from the disjoint union of reference simplices, identifying $\left|T^{\prime}\right|$ with $|T|$ in the preceding situation.

If $S$ is a subset of an affine space $V$, there are natural maps $\Phi_{T}:|T| \rightarrow V$, from reference simplices to $V$ :

$$
\Phi_{T}: x=\left(x_{i}\right)_{i \in T} \mapsto \sum_{i \in T}\left(x_{i}\right) i .
$$

We say that the simplex is non-degenerate if this map is injective. We say that the simplicial complex is non-degenerate if the induced map $|\mathcal{S}| \rightarrow V$ is injective.

More generally, simplicial cellular complexes are defined, replacing the affine maps (2.8) by Lipschitz isomorphisms onto their ranges, which are then called a simplicial cells or curved simplices. These maps should satisfy the relation $\Phi_{T} \circ i_{T T^{\prime}}=\Phi_{T^{\prime}}$ when $T^{\prime} \subseteq T$.

\subsection{Cochains}

Fix a cellular complex $(S, \mathcal{T})$. We suppose that for each $T \in \mathcal{T}$ of dimension $\geq 1$, the manifold $T$ has been oriented. The relative orientation of two cells $T$ and $T^{\prime}$ in $\mathcal{T}$, also called the incidence number, is denoted $o\left(T, T^{\prime}\right)$ and defined as follows. For any edge $e \in \mathcal{T}^{1}$ its vertices are ordered, from say $\dot{e}$ to $\ddot{e}$. Define $o(e, \dot{e})=-1$ and $o(e, \ddot{e})=1$. Concerning higher dimensional cells, fix $k \geq 1$. Given $T \in \mathcal{T}^{k+1}$ and $T^{\prime} \in \mathcal{T}^{k}$ such that $T^{\prime} \subseteq T$ we define $o\left(T, T^{\prime}\right)=1$ if $T^{\prime}$ is outward oriented compared with $T$ and $o\left(T, T^{\prime}\right)=-1$ if it is inward oriented. For all $T, T^{\prime} \in \mathcal{T}$ not covered by these definitions we put $o\left(T, T^{\prime}\right)=0$.

For each $k$, let $\mathcal{C}^{k}(\mathcal{T})$ denote the set of maps $c: \mathcal{T}^{k} \rightarrow \mathbb{R}$. Such maps associate a real number with each $k$-dimensional cell and are called $k$-cochains. The coboundary operator $\delta: \mathcal{C}^{k}(\mathcal{T}) \rightarrow \mathcal{C}^{k+1}(\mathcal{T})$ is defined by:

$$
(\delta c)_{T}=\sum_{T^{\prime} \in \mathcal{T}^{k}} o\left(T, T^{\prime}\right) c_{T^{\prime}}
$$

The space of $k$-cochains has a canonical basis indexed by $\mathcal{T}^{k}$. The coboundary operator is the operator whose canonical matrix is the incidence matrix $o$, indexed by $\mathcal{T}^{k+1} \times \mathcal{T}^{k}$. We remark that the coefficients in the sum can be non-zero only when $T^{\prime} \in \partial T \cap \mathcal{T}^{k}$. A fundamental property of $\delta$ is that $\delta \delta=0$ as a map $\mathcal{C}^{k}(\mathcal{T}) \rightarrow \mathcal{C}^{k+2}(\mathcal{T})$. In other words the family $\mathcal{C} \bullet(\mathcal{T})$ is a complex. It is called the called the cochain complex and represented by:

$$
0 \rightarrow \mathcal{C}^{0}(\mathcal{T}) \rightarrow \mathcal{C}^{1}(\mathcal{T}) \rightarrow \mathcal{C}^{2}(\mathcal{T}) \rightarrow \cdots
$$

When $S$ is a smooth manifold we denote by $\Omega^{k}(S)$ the space of smooth differential $k$-forms on $S$. Differential forms can be mapped to cochains as follows. Let $S$ be a manifold and $\mathcal{T}$ a cellular complex on $S$. For each $k$ we denote by $\rho^{k}: \Omega^{k}(S) \rightarrow \mathcal{C}^{k}(\mathcal{T})$ the de Rham map, which is defined by:

$$
\rho^{k}: u \mapsto\left(\int_{T} u\right)_{T \in \mathcal{T}^{k}} .
$$

As a consequence of Stokes theorem on each cell of $\mathcal{T}$ we have that for each $k$ 
the following diagram commutes:

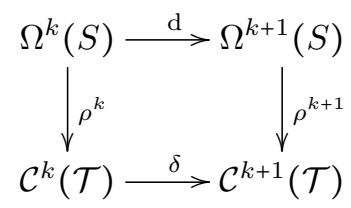

A celebrated theorem of de Rham states that the above map induces isomorphisms on cohomology groups. Whitney forms, that we shall soon encounter, were introduced in $[44,45]$ (relative to smooth partitions of unity) as a tool of proof, but have recently reappeared in numerical analysis (relative to piecewise affine partitions of unity). The next section introduces a general framework containing these forms as well as useful generalizations.

\section{$3 \quad$ Finite element systems}

\subsection{Definition}

For the purposes of analysis of partial differential equations and numerical methods, smooth differential forms are somewhat insufficient. Banach spaces of differential forms with various integrability conditions are used. For linear equations, $\mathrm{L}^{2}$ based Hilbert spaces are often enough.

For any cell $T$, we denote by $X^{k}(T)$ be the space of differential $k$-forms on $T$ which are $\mathrm{L}^{2}(T)$ and have exterior derivative, a priori defined as a current, in $\mathrm{L}^{2}(T)$. We let $X_{0}^{k}(T)$ be the closure of the subspace of $X^{k}(T)$ consisting of forms whose support is a compact subset of the interior of $T$. If $T^{\prime}$ is a face of $T$, let $i_{T T^{\prime}}: T^{\prime} \rightarrow T$ be the inclusion map. When $T^{\prime}$ has codimension 1 in $T$, the pullback of $k$-forms by $i_{T T^{\prime}}$ defines an operator which is bounded from $X^{k}(T)$ to the dual of $X_{0}^{\operatorname{dim} T^{\prime}-k}\left(T^{\prime}\right)$. It therefore makes sense to define:

$$
Y^{k}(T)=\left\{u \in X^{k}(T):\left.\forall T^{\prime} \in \mathcal{T} \quad T^{\prime} \subseteq T \Rightarrow u\right|_{T^{\prime}} \in X^{k}\left(T^{\prime}\right)\right\} .
$$

Definition 3.1. Suppose $\mathcal{T}$ is a cellular complex. For each $k \in \mathbb{N}$ and each $T \in \mathcal{T}$ we suppose we are given a space $E^{k}(T) \subseteq Y^{k}(T)$, called a differential $k$-element on $T$. The following conditions should be satisfied:

- If $i_{T T^{\prime}}: T^{\prime} \subseteq T$ is an inclusion of cells, pullback induces a map $i_{T T^{\prime}}^{\star}$ : $E^{k}(T) \rightarrow E^{k}\left(T^{\prime}\right)$.

- The exterior derivative induces maps $\mathrm{d}: E^{k}(T) \rightarrow E^{k+1}(T)$, for all cells $T \in \mathcal{T}$ and all $k$.

Such a family of elements is called an element system.

A differential element is said to be finite if it is finite dimensional. A finite element system, abbreviated FES in the following, is an element system in which all the elements are finite.

For any subcomplex $\mathcal{T}^{\prime}$ of $\mathcal{T}$ we define $E^{k}\left(\mathcal{T}^{\prime}\right)$ as follows :

$$
E^{k}\left(\mathcal{T}^{\prime}\right)=\left\{u \in \bigoplus_{T \in \mathcal{T}^{\prime}} E^{k}(T): \forall T,\left.T^{\prime} \in \mathcal{T} \quad T^{\prime} \subseteq T \Rightarrow u_{T}\right|_{T^{\prime}}=u_{T^{\prime}}\right\} .
$$


An FES over a cellular complex is an inverse system of complexes. To inclusion of cells $T^{\prime} \subseteq T$, correspond pullback operators $i_{T T^{\prime}}^{\star}$, which are morphisms of complexes with some obvious properties:

$$
\begin{aligned}
i_{T T}^{\star} & =\mathrm{id}, \\
i_{T^{\prime} T^{\prime \prime}}^{\star} \circ i_{T T^{\prime}}^{\star} & =i_{T T^{\prime \prime}}^{\star}, \text { when } T^{\prime \prime} \subseteq T^{\prime} \subseteq T .
\end{aligned}
$$

The space $E^{\bullet}\left(\mathcal{T}^{\prime}\right)$ defined above is an inverse limit of this system and is determined by this property up to unique isomorphism. For a cell $T \in \mathcal{T}$, its collection of subcells is the cellular complex $\tilde{T}$. Applied to this case, the above definition of $E^{k}(\tilde{T})$ gives a space canonically isomorphic to $E^{k}(T)$, so that we can identify $E^{k}(\tilde{T})=E^{k}(T)$.

Elements of $E^{k}\left(\mathcal{T}^{\prime}\right)$ may be regarded as differential forms defined cell-wise, which have a partial continuity property across interfaces between cells, in the form of equal pullbacks.

Applied to $\mathcal{T}$ this will provide spaces $E^{k}(\mathcal{T})$ suitable for Galerkin and Petrov-Galerkin discretizations of PDEs expressed in terms of exterior derivatives.

Also of importance is the application of the above construction to the boundary $\partial T$ of a cell $T$, considered as a cellular complex consisting of all subcells of $T$ except $T$ itself. Considering $\partial T$ as a cellular complex (not only a subset of $T$ ) we denote the constructed inverse limit by $E^{k}(\partial T)$. If $i: \partial T \rightarrow T$ denotes the inclusion map, the pullback by $i$ defines a map $i^{\star}: E^{k}(T) \rightarrow E^{k}(\partial T)$ which we denote by $\partial$ and call restriction.

Given an element system $E$ on a cellular complex $\mathcal{T}$, we consider now the following conditions:

- Extensions. For each $T \in \mathcal{T}$ and $k \in \mathbb{N}$, restriction $\partial: E^{k}(T) \rightarrow E^{k}(\partial T)$ is onto.

- Exactness. The following sequence is exact for each $T \in \mathcal{T}$ :

$$
0 \rightarrow \mathbb{R} \rightarrow E^{0}(T) \rightarrow E^{1}(T) \rightarrow \cdots \rightarrow E^{\operatorname{dim} T}(T) \rightarrow 0 .
$$

Definition 3.2. We will say that an element system is compatible if the two conditions above hold.

The first surjectivity condition can be written symbolically $\partial E^{k}(T)=E^{k}(\partial T)$. We denote also:

$$
\begin{aligned}
E_{0}^{k}(T) & =\operatorname{ker} \partial: E^{k}(T) \rightarrow E^{k}(\partial T), \\
& =E^{k}(T) \cap X_{0}^{k}(T) .
\end{aligned}
$$

The latter equality requires some work.

The following is Theorem 3.1 of [16], see also Proposition 5.16 in [20], and is comparable to well-known so called piecewise de Rham theorems, see e.g. [25].

Proposition 3.1. If $E$ is a compatible finite element system, the de Rham map $\rho^{\bullet}: E^{\bullet}(\mathcal{T}) \rightarrow \mathcal{C} \bullet(\mathcal{T})$ induces isomorphisms on cohomology groups.

Compatible FES also have fairly local bases, see Remark 4.2. 


\subsection{Examples}

We now provide some examples of element systems, all of which are compatible. Example 3.1. The spaces $Y^{k}(T)$ themselves define an element system. It is far from finite.

On the other hand the spaces $X^{k}(T)$ do not constitute an element system. This family of spaces is stable under the exterior derivative, but not under restriction from cells to subcells.

Example 3.2. In dimension 1, consider a cellular complex with notations as in Example 2.1. In the following examples $E^{0}\left(\left\{x_{i}\right\}\right)=\mathbb{R}^{\left\{x_{i}\right\}} \approx \mathbb{R}$ for each $i$.

- Piecewise polynomials. The space of polynomials of degree at most $p$ is denoted $\mathcal{P}_{p}$. Pick an integer $p_{i} \geq 1$ for each $i \in[[0, n-1]$. Define:

$$
\begin{aligned}
& E^{0}\left(\left[x_{i}, x_{i}+1\right]\right)=\mathcal{P}_{p_{i}}, \\
& E^{1}\left(\left[x_{i}, x_{i}+1\right]\right)=\mathcal{P}_{p_{i}-1} \mathrm{~d} x .
\end{aligned}
$$

Here, restriction of the polynomials to the interval is implicit.

- Piecewise exponentials. Pick a real $r_{i} \neq 0$ for each $i \in \llbracket[0, n-1]$. Define:

$$
\begin{aligned}
& E^{0}\left(\left[x_{i}, x_{i}+1\right]\right)=\left\{x \mapsto c_{0}+c_{1} \exp \left(r_{i} x\right): c_{0}, c_{1} \in \mathbb{R}\right\}, \\
& E^{1}\left(\left[x_{i}, x_{i}+1\right]\right)=\left\{x \mapsto c \exp \left(r_{i} x\right) \mathrm{d} x: c \in \mathbb{R}\right\} .
\end{aligned}
$$

- Splines, see [43], can also be accommodated. Inserting knots in cells $\left[x_{i}, x_{i+1}\right]$ is fine, but between cells one has mere continuity in $E^{0}$, and no continuity in $E^{1}$.

- More generally take, on each interval, for $E^{0}$, a space of functions containing at least a constant function and one with different values at the extremities, and for $E^{1}$ the space of derivatives.

Example 3.3. In the situation of Example 2.2, suppose that one has differential elements $E^{k}(U)$ for $U \in \mathcal{U}$ and $F^{k}(V)$ for $V \in \mathcal{V}$, both forming systems according to Definition 3.1. It is then possible to define a natural notion of tensor product of $E$ and $F$, as an FES on the product complex of $\mathcal{U}$ and $\mathcal{V}$, see [20] p. 77-79. As one might expect, if $E$ and $F$ are compatible, in the sense of Definition 3.2, then so is their tensor product.

This can be combined with the preceding Examples to define FES on products of intervals.

Example 3.4. Let $V$ be a finite dimensional vector space. We denote by $\mathcal{P}_{p}(V)$ the space of polynomials on $V$ of degree at most $p$, by $\Lambda^{k}(V)$ the space of alternating $k$-linear maps $V^{k} \rightarrow \mathbb{R}$ and by $\mathcal{P} \Lambda_{p}^{k}(V)$ the space of differential $k$-forms on $V$ which are polynomials of degree at most $p$.

Denote the Koszul operator by $\kappa$. It is the contraction of differential forms by the identity, interpreted as a vectorfield:

$$
(\kappa u)_{x}\left(\xi_{1}, \ldots, \xi_{k}\right)=u_{x}\left(x, \xi_{1}, \ldots, \xi_{k}\right) .
$$


Alternatively one can use the Poincaré operator associated with the canonical homotopy from the identity to the null-map. Let $\mathcal{T}$ be a simplicial complex. Define, for any non-zero $p \in \mathbb{N}$, any simplex $T \in \mathcal{T}$ and any $k \in \mathbb{N}$ :

$$
\begin{aligned}
\Lambda_{p}^{k}(T) & =\left\{u \in \mathcal{P} \Lambda_{p}^{k}(T): \kappa u \in \mathcal{P} \Lambda_{p}^{k-1}(T)\right\}, \\
& =\mathcal{P} \Lambda_{p-1}^{k}(T)+\kappa \mathcal{P} \Lambda_{p-1}^{k+1}(T) .
\end{aligned}
$$

For fixed $p$, we call this the trimmed polynomial finite element system of order $p$.

This construction, due to [36] for $\mathbb{R}^{2},[34]$ for vector fields in $\mathbb{R}^{3}$, and [27] for differential forms, is thoroughly treated in [3]. The case $p=1$ corresponds to constructs in $[44,45]$, called Whitney forms, as pointed out in [7].

Whitney forms are defined as follows. For a given simplicial mesh $\mathcal{T}$, let $\lambda_{i}$ denote the barycentric coordinate map associated with vertex $i$. It is the unique continuous and piecewise affine function taking the value 1 at vertex $i$ and the value 0 at other vertices $j \in \mathcal{T}^{0}$. To a simplex $T \in \mathcal{T}^{k}$ of dimension $k \geq 1$ one attaches the $k$-form $\lambda_{T}$ defined by:

$$
\lambda_{T}=k ! \sum_{i=0}^{k}(-1)^{i} \lambda_{i} \mathrm{~d} \lambda_{0} \wedge \cdots\left(\mathrm{d} \lambda_{i}\right)^{\wedge} \cdots \wedge \mathrm{d} \lambda_{k} .
$$

Here, the vertices of $T$ are labelled $\{0, \ldots, k\}$, respecting the orientation of $T$. The superscript $(\cdot)^{\wedge}$ signifies omission of this term. The $\lambda_{T}$ for $T \in \mathcal{T}^{k}$ constitute a basis for $\Lambda_{1}^{k}(\mathcal{T})$.

It was usual to start the indexing in (3.13) at $p=0$ but, as remarked in the preprint of [15], the advantage of letting the lowest order be $p=1$, is that the wedge product induces maps:

$$
\wedge: \Lambda_{p_{0}}^{k_{0}}(T) \times \Lambda_{p_{1}}^{k_{1}}(T) \rightarrow \Lambda_{p_{0}+p_{1}}^{k_{0}+k_{1}}(T) .
$$

In words, the wedge product respects the grading in $k$ and the filtering in $p$.

Example 3.5. A convenient way of constructing a compatible FES on a cellular complex $\mathcal{T}$ is to take a refinement $\mathcal{T}^{\prime}$ on which one has a compatible FES $E^{k}(T)$, and consider this as an FES on $\mathcal{T}$. Namely, if $T \in \mathcal{T}$, consider $T$ as a cellular subcomplex $\tilde{T}$ of $\mathcal{T}^{\prime}$ and define $F^{k}(T)=E^{k}(\tilde{T})$. The Galerkin spaces associates with $E$ and $F$ are identical, even though they are different as FES. The versatility of such composite finite elements has already been put to good use, perhaps most famously in the Clough-Tocher element of class $C^{1}(S)$.

Example 3.6. For an interpretation of so-called $h p$ finite elements in this framework, see Example 5.24 p. 79 in [20].

Remark 3.1. This point of view on finite elements is at variance with Ciarlet's definition, as provided, for instance, in [22] $\$ 10$.

- Firstly, we consider fields not just on cells of maximal dimension but also on all their faces.

- Secondly, we consider at once differential forms of all degrees, rather that say just functions or just vector fields.

- Thirdly, so called degrees of freedom are not part of the definition, instead inter-element continuity is enforced by conditions on pullbacks to faces. 
Our definition is more restrictive in the sense that we have not accommodated say finite elements for matrix fields such as those of elasticity or those with higher order inter-element continuity such as Hermite polynomials. On the other hand it is tailored to account for the successes of the elements defined in Example 3.4.

\section{Upwinding in FES}

\subsection{Locally harmonic forms}

Let $T$ be a cell where, for each $k, E^{k}(T)$ is equipped with a scalar product $b$. Orthogonality with respect to $b$ will be denoted $\perp$. We say that a $k$-form $u$ on $T$ is E-harmonic if:

$$
\mathrm{d} u \perp \mathrm{d} E_{0}^{k}(T) \text { and } u \perp \mathrm{d} E_{0}^{k-1}(T) .
$$

One can for instance take $b$ to be the $\mathrm{L}^{2}(T)$ scalar product on differential forms, associated with some Riemannian metric. Denote by $\mathrm{d}^{\star}$ the formal adjoint of $d$ with respect to this scalar product. The continuous analogue of the above condition (4.1) is:

$$
\mathrm{d}^{\star} \mathrm{d} u=0 \text { and } \mathrm{d}^{\star} u=0 .
$$

From the other point of view, (4.1) is the Galerkin variant of (4.2).

Let $E$ be a finite element system on $\mathcal{T}$. Define a finite element system $\stackrel{\circ}{E}$ by:

$$
\stackrel{\circ}{E}^{k}(T)=\left\{u \in E^{k}(T):\left.\forall T^{\prime} \in \mathcal{T} \quad T^{\prime} \subseteq T \Rightarrow u\right|_{T^{\prime}} \text { is } E \text {-harmonic }\right\} .
$$

We say that $\stackrel{\circ}{E}$ is the subsystem of $E$ of locally harmonic forms.

The following was essentially proved in [16].

Proposition 4.1. If $E$ is a compatible FE system then $\stackrel{\circ}{E}$ is a compatible $F E$

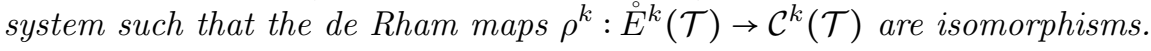

Example 4.1. This construction generalizes [30], in which div-conforming finite element vector fields are defined on polyhedral meshes in $\mathbb{R}^{3}$.

Remark 4.1. Since $\mathcal{C}^{k}(\mathcal{T})$ has a canonical basis, the de Rham map determines a corresponding canonical basis of $\stackrel{\circ}{E}^{k}(\mathcal{T})$. Its elements can be constructed by recursive harmonic extension. Explicitly, to construct the $k$-form $u_{T}$ attached to a given $T \in \mathcal{T}^{k}$, define $\left.u_{T}\right|_{T}$ to be the unique element of $E^{k}(T)$ orthogonal to $\mathrm{d} E_{0}^{k-1}(T)$ and with integral 1. For $T^{\prime} \in \mathcal{T}^{k}$, different from $T$, set $\left.u_{T}\right|_{T^{\prime}}=0$. Supposing $u$ has been defined on the $l$-skeleton of $\mathcal{T}$ (constituted by cells of dimension at most $l), u$ is extended to the $(l+1)$-skeleton through harmonic extension to $(l+1)$-dimensional cells, as defined by $(4.1)$.

Remark 4.2. More generally it is possible, for any compatible FES $E$, to define isomorphisms:

$$
\bigoplus_{T \in \mathcal{T}} E_{0}^{k}(T) \rightarrow E^{k}(\mathcal{T})
$$

through a procedure of recursive harmonic extension, see Proposition 3.14 in [17] (also Proposition 3.1 and Remark 3.3 in [16]). More general isomorphisms are constructed in [4]. 
Example 4.2. Start with a fine cellular complex, and coarsen it by agglomerating cells, as in Example 3.5. An FES on the fine mesh yields an FES on the coarse mesh, of which one can consider the subsystem of locally harmonic forms. The construction can be applied recursively, agglomerating cells at each step, and taking at a given level the locally harmonic fields of the refined level. This yields nested spaces that can be used for multilevel preconditioning [35].

Example 4.3. On simplices, lowest order trimmed polynomial differential forms are locally harmonic in the sense of (4.2), with respect to the $\mathrm{L}^{2}(T)$ product associated with any Euclidean metric (a Riemannian metric which is constant on $T$ ), as pointed out in $\S 4.1$ of [16]. Whitney forms, as defined by (3.15), provide an explicit solution to the problem of recursive harmonic extension.

Example 4.4. For a given complex $\mathcal{T}$, consider its barycentric refinement $\mathcal{T}^{\prime}$ (assuming it is well defined), and reassemble the simplices of $\mathcal{T}^{\prime}$ to form the dual $\mathcal{T}^{\star}$ of $\mathcal{T}$. Take lowest order trimmed polynomial differential forms on $\mathcal{T}^{\prime}$. Consider them once as an FES on $\mathcal{T}$ and once as an FES on $\mathcal{T}^{\star}$, as in Example 3.5. For these two FES consider their subsystems of locally harmonic forms. At least in some cases, one can prove that these two subsystems are in duality, providing a finite element analogue of Hodge duality, which has applications to preconditioning [14]. For a tentative application to fluid dynamics, see Example 5.30 in $[20]$.

Remark 4.3. Local harmonicity appears to behave naturally with respect to the tensor products of Example 3.3.

\subsection{Application to upwinding}

We now apply the construction of locally harmonic forms to obtain a generalization of Section 1 to multidimensional problems, extending also, for instance, [12]. We have in mind applications to fluid dynamics. A model problem for this situation is the following. Consider a bounded domain $S$ in a Euclidean space, $\alpha>0$, a divergence free vector field $V$ on $S$ and also a function $f$ on $S$. Consider the equation for a scalar field $u$ on $S$ :

$$
\begin{aligned}
-\alpha \Delta u+V \cdot \operatorname{grad} u & =f \text { in } S, \\
\left.u\right|_{\partial S} & =0 .
\end{aligned}
$$

The variational form of this equation is:

$$
u \in X_{0}^{0}(S), \forall v \in X_{0}^{0}(S) \int \operatorname{grad} u \cdot(\alpha \operatorname{grad} v+V v)=\int f v .
$$

One is interested in the asymptotic behavior of $u$ for small positive $\alpha$. One is also interested in similar problems for vector fields. Typically the flow field itself satisfies an equation of this type. More generally, analogous equations for differential forms are of interest. We are going to design Petrov-Galerkin methods of the form (1.17) for this purpose.

Consider a cellular complex and a large compatible finite element system on it, obtained for instance by considering trimmed polynomial differential forms on a simplicial refinement, as in Example 3.4. We let one space (say the trial space) consist of locally harmonic forms for the standard $\mathrm{L}^{2}$ product, deduced from the Euclidean metric. For the other space (say the test space) we use 
locally harmonic forms for a weighted $\mathrm{L}^{2}$ product. To motivate our choice of weight we introduce some machinery.

Let $\beta$ be a one-form on $S$. The associated covariant exterior derivative is:

$$
\mathrm{d}_{\beta}: u \mapsto \mathrm{d} u+\beta \wedge u .
$$

These operators do not form a complex but we have:

$$
\mathrm{d}_{\beta} \mathrm{d}_{\beta} u=(\mathrm{d} \beta) \wedge u .
$$

In gauge theory (see [29]) the term $\mathrm{d} \beta$ is called curvature. Supposing that $\beta=\mathrm{d} \phi$ for a function $\phi$ we have:

$$
\mathrm{d}_{\beta}=\exp (-\phi) \mathrm{d} \exp (\phi) u .
$$

One says that $u \mapsto \exp (\phi) u$ is a gauge transformation.

We denote by $\mathrm{d}_{\beta}^{\star}$ the formal adjoint of $\mathrm{d}_{\beta}$ with respect to the $\mathrm{L}^{2}$ scalar product on differential forms. When $\beta=\mathrm{d} \phi$ we have:

$$
\mathrm{d}_{\beta}^{\star} u=\exp (\phi) \mathrm{d}^{\star} \exp (-\phi) .
$$

A natural generalization of $(4.2)$ is then:

$$
\mathrm{d}_{\beta}^{\star} \mathrm{d} u=0 \text { and } \mathrm{d}_{\beta}^{\star} u=0,
$$

which can be written:

$$
\mathrm{d}^{\star} \exp (-\phi) \mathrm{d} u=0 \text { and } \mathrm{d}^{\star} \exp (-\phi) u=0 .
$$

Returning to (4.5) we suppose $S$ is a domain in a Euclidean space. We fix a cellular complex $\mathcal{T}$ on $S$, with flat cells, and a large compatible FE system $E$ on it, as indicated above. This means that $E_{0}^{k}(T)$ should have large enough dimension, to accommodate a variety of differential forms. We construct two spaces of locally harmonic forms deduced from $E$, distinguished by the choice of scalar product $b$ defining orthogonality in (4.1). For one (the trial space) take $b$ to be the standard $\mathrm{L}^{2}$ scalar product. For the other (the test space) we introduce the 1-form $\beta$ defined by:

$$
\beta_{x}(\xi)=V(x) \cdot \xi
$$

We choose for each $T \in \mathcal{T}$, a constant approximation $\beta_{T}$ of the pullback of $\beta$ to $T$. Let $\phi_{T}$ be an affine function such that $\mathrm{d} \phi_{T}=\beta_{T}$. One can determine it completely for instance by imposing zero mean on $T$. To mimick (4.13) we use the scalar product:

$$
b(u, v)=\int_{T} \exp \left(\phi_{T} / \alpha\right) u \cdot v,
$$

to define the locally harmonic functions. For explicitness, recall that the local trial functions $u \in \dot{E}^{k}(T)$ should then satisfy the equations:

$$
\begin{aligned}
& \forall v \in E_{0}^{k}(T) \quad \int \exp \left(\phi_{T} / \alpha\right) \mathrm{d} u \cdot \mathrm{d} v=0, \\
& \forall v \in E_{0}^{k-1}(T) \quad \int \exp \left(\phi_{T} / \alpha\right) u \cdot \mathrm{d} v=0 .
\end{aligned}
$$


The canonical basis of the test space, as defined by Remark 4.1, will then be upwinded, compared with the canonical basis of the trial space. To get an idea of the shape of upwinded basis functions we can remark that when $v$ is a constant differential $k$-form on $T, u=\exp \left(-\phi_{T} / \alpha\right) v$ satisfies the equations $(4.16,4.17)$, whatever element system is used.

Example 4.5. In dimension 1 we recover the upwinded basis functions defined in Section 1, from the infinite dimensional element system $Y$. These are "true" locally harmonic functions, with respect to an exponential weight. In higher dimensions one cannot hope to have explicit formulas for these, which is why we have introduced discrete analogues. The challenge was to get a discrete analogue where basic properties, such as local sequence exactness with respect to the exterior derivative, are satisfied.

Remark 4.4. There is at least one case in which one can get an explicit formula for the upwinded differential forms, namely the extension of 0 -forms from vertices to the 1 -skeleton. In some cases this is all one needs. Let $\mathcal{T}$ be a simplicial complex. Define a coefficient $\mu_{e}$ attached to the edge $e \in \mathcal{T}^{1}$, with vertices $i$ and $j$, by:

$$
\mu_{e}=-\int_{S} \mathrm{~d} \lambda_{i} \cdot \mathrm{d} \lambda_{j}
$$

We suppose the simplices are such that $\mu_{e} \geq 0$. For Whitney 1-forms $u, v \in \Lambda_{1}^{1}(\mathcal{T})$ one then has an estimate of the form:

$$
\left|\int u \cdot v-\sum_{e} \mu_{e}\left(\rho^{1} u\right)_{e}\left(\rho^{1} v\right)_{e}\right| \leq C h\left(\|u\|_{\mathrm{L}^{2}}\|\mathrm{~d} v\|_{\mathrm{L}^{2}}+\|\mathrm{d} u\|_{\mathrm{L}^{2}}\|v\|_{\mathrm{L}^{2}}\right),
$$

where, we sum over edges $e \in \mathcal{T}^{1}$ and $h$ represents the largest diameter of a simplex of $\mathcal{T}$, see [26] (or [19]). This indicates that the discrete sum on the left hand side is a good approximation of the integral.

Define, on $X_{0}^{0}(S) \times X_{0}^{0}(S)$ :

$$
a(u, v)=\int \operatorname{grad} u \cdot(\alpha \operatorname{grad} v+V v),
$$

and its approximation ( $\rho$ is the de Rham map):

$$
a_{h}(u, v)=\sum_{e} \mu_{e}\left(\rho^{1} \mathrm{~d} u\right)_{e}\left(\rho^{1}\left(\alpha \mathrm{d} v+\beta_{e} v\right)\right)_{e}
$$

Letting $u \in \Lambda_{1}^{0}(\mathcal{T})$ and $v$ be defined at vertices and upwinded on edges according to (4.16) with $E=Y$, we recover the scheme of [46].

Remark 4.5. Residual Free Bubbles (see e.g. [10]) on the other hand, from which the Streamline Upwind Petrov Galerkin method [13] can be deduced, works by extending standard finite elements on cells of maximal dimension only, by the whole space $X_{0}^{k}(T)$, and reducing the resulting equation to a modifed one on the original space in special circumstances.

\section{$5 \quad$ Further topics}

High order upwinding. Let $E$ be a large compatible FES, providing workspace, and let $F$ be a compatible FES that we wish to upwind, within $E$. For any function $\phi$ defined on the simplexes of $\mathcal{T}$, we denote by $b_{\phi}$ the weigthed $\mathrm{L}^{2}$ product 
of forms on $T$ :

$$
b_{\phi}(u, v)=\int_{T} \exp \left(\phi_{T}\right) u \cdot v .
$$

We define:

$$
\begin{aligned}
G^{k}(T)=\{ & \left\{u \in E^{k}(T): \forall T^{\prime} \subseteq T \exists v \in F_{0}^{k}\left(T^{\prime}\right)\right. \\
& \forall w \in E_{0}^{k}\left(T^{\prime}\right) \quad b_{\phi}(\mathrm{d} u, \mathrm{~d} w)=b_{0}(\mathrm{~d} v, \mathrm{~d}(\exp (\phi) w)), \\
& \left.\forall w \in E_{0}^{k-1}\left(T^{\prime}\right) \quad b_{\phi}(u, \mathrm{~d} w)=b_{0}(v, \mathrm{~d}(\exp (\phi) w))\right\}
\end{aligned}
$$

Then $G$ defines a compatible FES, of the same dimension as $F$, which appears to be locally upwinded version of it (scaling $\phi$ by $\alpha$ ).

Minimal FES. The framework of FES can also be applied to construct a minimal compatible FES containing certain prespecified differential forms, see [18]. The trimmed polynomial finite element system of order $p$ is a minimal compatible one containing polynomial differential forms of order $p-1$.

Vector bundles. The covariant exterior derivative defined by (4.8) corresponds to a very special choice of vector bundle, namely one with fiber $\mathbb{R}$ and gauge group $\mathbb{R}_{+}$consisting of positive reals under multiplication. A somewhat more elaborate variant uses fibers $\mathbb{C}$ and gauge group $\mathbb{U}(1)$ consisting of complex numbers with modulus 1 . For an extension of the present theory to this case, with applications to wave equations of Helmholtz type, see Example 5.32 in $[20]$.

Analysis. At present we are quite far from a satisfactory analysis of upwinded FES. Even for one dimensional problems there are recent interesting developments, e.g. [40]. For standard FES, such as trimmed polynomial differential forms (Example 3.4), a powerful tool for convergence proofs is the construction of projections that commute with the exterior derivative, yet are uniformly bounded with respect to $\mathrm{L}^{2}$ norms, $[42,15,3,21]$. The important property of these examples is that they are scale invariant FES, and the analysis has been carried over to this setting in [20] §5.2-4. Various $\mathrm{L}^{p}$ estimates are obtained, yielding discrete Sobolev injections and translation estimates. However, upwinded FES are not scale invariant, since they take into account certain length scales, such as the thickness of the boundary layers.

\section{References}

[1] D. N. de G. Allen and R. V. Southwell. Relaxation methods applied to determine the motion, in two dimensions, of a viscous fluid past a fixed cylinder. Quart. J. Mech. Appl. Math., 8:129-145, 1955.

[2] D. N. Arnold. Differential complexes and numerical stability. In Proceedings of the International Congress of Mathematicians, Vol. I (Beijing, 2002), pages 137-157, Beijing, 2002. Higher Ed. Press.

[3] D. N. Arnold, R. S. Falk, and R. Winther. Finite element exterior calculus, homological techniques, and applications. Acta Numer., 15:1-155, 2006. 
[4] D. N. Arnold, R. S. Falk, and R. Winther. Geometric decompositions and local bases for spaces of finite element differential forms. Comput. Methods Appl. Mech. Engrg., 198(21-26):1660-1672, 2009.

[5] D. N. Arnold, R. S. Falk, and R. Winther. Finite element exterior calculus: from Hodge theory to numerical stability. Bull. Amer. Math. Soc. (N.S.), $47(2): 281-354,2010$.

[6] P. B. Bochev and J. M. Hyman. Principles of mimetic discretizations of differential operators. In Compatible spatial discretizations, volume 142 of IMA Vol. Math. Appl., pages 89-119. Springer, New York, 2006.

[7] A. Bossavit. Mixed finite elements and the complex of Whitney forms. In The mathematics of finite elements and applications, VI (Uxbridge, 1987), pages 137-144. Academic Press, London, 1988.

[8] R. Bott and L. W. Tu. Differential forms in algebraic topology, volume 82 of Graduate Texts in Mathematics. Springer-Verlag, New York, 1982.

[9] F. Brezzi and M. Fortin. Mixed and hybrid finite element methods, volume 15 of Springer Series in Computational Mathematics. Springer-Verlag, New York, 1991.

[10] F. Brezzi, T. J. R. Hughes, L. D. Marini, A. Russo, and E. Süli. A priori error analysis of residual-free bubbles for advection-diffusion problems. SIAM J. Numer. Anal., 36(6):1933-1948, 1999.

[11] F. Brezzi, K. Lipnikov, and M. Shashkov. Convergence of the mimetic finite difference method for diffusion problems on polyhedral meshes. SIAM J. Numer. Anal., 43(5):1872-1896 (electronic), 2005.

[12] F. Brezzi, L. D. Marini, and P. Pietra. Two-dimensional exponential fitting and applications to drift-diffusion models. SIAM J. Numer. Anal., 26(6):1342-1355, 1989.

[13] A. N. Brooks and T. J. R. Hughes. Streamline upwind/Petrov-Galerkin formulations for convection dominated flows with particular emphasis on the incompressible Navier-Stokes equations. Comput. Methods Appl. Mech. Engrg., 32(1-3):199-259, 1982. FENOMECH '81, Part I (Stuttgart, 1981).

[14] A. Buffa and S. H. Christiansen. A dual finite element complex on the barycentric refinement. Math. Comp., 76(260):1743-1769 (electronic), 2007.

[15] S. H. Christiansen. Stability of Hodge decompositions in finite element spaces of differential forms in arbitrary dimension. Numer. Math., 107(1):87-106, 2007.

[16] S. H. Christiansen. A construction of spaces of compatible differential forms on cellular complexes. Math. Models Methods Appl. Sci., 18(5):739-757, 2008.

[17] S. H. Christiansen. Foundations of finite element methods for wave equations of Maxwell type. In Applied Wave Mathematics, pages 335-393. Springer, Berlin Heidelberg, 2009. 
[18] S. H. Christiansen. Éléments finis mixtes minimaux sur les polyèdres. $C$. R. Math. Acad. Sci. Paris, 348(3-4):217-221, 2010.

[19] S. H. Christiansen and T. G. Halvorsen. A gauge invariant discretization on simplicial grids of the Schrödinger eigenvalue problem in an electromagnetic field. SIAM Journal on Numerical Analysis, 49(1):331-345, 2011.

[20] S. H. Christiansen, H. Z. Munthe-Kaas, and B. Owren. Topics in structurepreserving discretization. Acta Numerica, 20:1-119, 2011.

[21] S. H. Christiansen and R. Winther. Smoothed projections in finite element exterior calculus. Math. Comp., 77(262):813-829, 2008.

[22] P. G. Ciarlet. Basic error estimates for elliptic problems. In Handbook of numerical analysis, Vol. II, Handb. Numer. Anal., II, pages 17-351. NorthHolland, Amsterdam, 1991.

[23] J. Dodziuk and V. K. Patodi. Riemannian structures and triangulations of manifolds. J. Indian Math. Soc. (N.S.), 40(1-4):1-52 (1977), 1976.

[24] J. Droniou, R. Eymard, T. Gallouët, and R. Herbin. A unified approach to mimetic finite difference, hybrid finite volume and mixed finite volume methods. Math. Models Methods Appl. Sci., 20(2):265-295, 2010.

[25] P. A. Griffiths and J. W. Morgan. Rational homotopy theory and differential forms, volume 16 of Progress in Mathematics. Birkhäuser Boston, Mass., 1981.

[26] Y. Haugazeau and P. Lacoste. Condensation de la matrice masse pour les éléments finis mixtes de $H$ (rot). C. R. Acad. Sci. Paris Sér. I Math., 316(5):509-512, 1993.

[27] R. Hiptmair. Canonical construction of finite elements. Math. Comp., 68(228):1325-1346, 1999.

[28] R. Hiptmair. Finite elements in computational electromagnetism. Acta Numer., 11:237-339, 2002.

[29] S. Kobayashi and K. Nomizu. Foundations of differential geometry. Vol I. Interscience Publishers, a division of John Wiley \& Sons, New York-Lond on, 1963.

[30] Y. Kuznetsov and S. Repin. Convergence analysis and error estimates for mixed finite element method on distorted meshes. J. Numer. Math., 13(1):33-51, 2005.

[31] P. Monk. Finite element methods for Maxwell's equations. Numerical Mathematics and Scientific Computation. Oxford University Press, New York, 2003.

[32] K. W. Morton. Numerical solution of convection-diffusion problems, volume 12 of Applied Mathematics and Mathematical Computation. Chapman \& Hall, London, 1996. 
[33] K. W. Morton. The convection-diffusion Petrov-Galerkin story. IMA J. Numer. Anal., 30(1):231-240, 2010.

[34] J.-C. Nédélec. Mixed finite elements in $\mathbf{R}^{3}$. Numer. Math., 35(3):315-341, 1980.

[35] J. E. Pasciak and P. S. Vassilevski. Exact de Rham sequences of spaces defined on macro-elements in two and three spatial dimensions. SIAM J. Sci. Comput., 30(5):2427-2446, 2008.

[36] P.-A. Raviart and J. M. Thomas. A mixed finite element method for 2nd order elliptic problems. In Mathematical aspects of finite element methods (Proc. Conf., Consiglio Naz. delle Ricerche (C.N.R.), Rome, 1975), pages 292-315. Lecture Notes in Math., Vol. 606. Springer, Berlin, 1977.

[37] J. E. Roberts and J.-M. Thomas. Mixed and hybrid methods. In Handbook of numerical analysis, Vol. II, Handb. Numer. Anal., II, pages 523-639. North-Holland, Amsterdam, 1991.

[38] H.-G. Roos, M. Stynes, and L. Tobiska. Robust numerical methods for singularly perturbed differential equations, volume 24 of Springer Series in Computational Mathematics. Springer-Verlag, Berlin, second edition, 2008. Convection-diffusion-reaction and flow problems.

[39] A. Russo. Streamline-upwind Petrov/Galerkin method (SUPG) vs residualfree bubbles (RFB). Comput. Methods Appl. Mech. Engrg., 195(1316):1608-1620, 2006.

[40] G. Sangalli. Robust a-posteriori estimator for advection-diffusion-reaction problems. Math. Comp., 77(261):41-70 (electronic), 2008.

[41] D.L. Scharfetter and H.K. Gummel. Large-signal analysis of a silicon read diode oscillator. Electron Devices, IEEE Transactions on, 16(1):64 - 77, jan 1969.

[42] J. Schöberl. A posteriori error estimates for Maxwell equations. Math. Comp., 77(262):633-649, 2008.

[43] L. L. Schumaker. Spline functions: basic theory. Cambridge Mathematical Library. Cambridge University Press, Cambridge, third edition, 2007.

[44] A. Weil. Sur les théorèmes de de Rham. Comment. Math. Helv., 26:119$145,1952$.

[45] H. Whitney. Geometric integration theory. Princeton University Press, Princeton, N. J., 1957.

[46] J. Xu and L. Zikatanov. A monotone finite element scheme for convectiondiffusion equations. Math. Comp., 68(228):1429-1446, 1999. 